\title{
The NOLA Project
}

\section{Des photoreportages au service de l'éducation inclusive des filles}

\author{
Manuela Clément-Frencia \\ Membre Fondatrice \\ The NOLA Project, Montréal, Québec, nolaprojectorg@gmail.com
}

\begin{abstract}
Résumé
Nombreuses sont les filles dans le monde qui souhaitent aller à l'école et réaliser leurs rêves. Pourtant, 263 millions d'enfants, d'adolescents et de jeunes à travers le monde - soit un sur cinq - ne sont pas scolarisés (UNESCO, 2018). Bien que l'écart se réduise entre filles et garçons, en 2016, 18,5\% des filles d'âge scolaire n'ont pas accès à une éducation. Défenseur des droits des enfants et du droit à l'éducation, The NOLA Project sensibilise les publics à l'éducation des filles avec des histoires inspirantes et des images puissantes dans un contexte d'autonomisation, d'inclusion et de réussite. Nous réalisons des reportages à forte valeur sociale pour témoigner de l'effet positif et durable qu'ont les programmes d'éducation sur la réussite des jeunes filles et sur la transformation des communautés.
\end{abstract}

Mots-clés : filles, éducation, photo reportage, exposition 


\section{Introduction}

Leyla a 18 ans. Elle est Somalienne. Elle habite dans le camp de réfugiés de Kakuma avec sa mère et ses deux jeunes frères. Leyla fréquente l'école primaire de BahrEl-Naam où elle suit des cours de rattrapage car elle a dû interrompre sa scolarité à cause de la guerre dans son pays d'origine. Elle participe également à des sessions de leadership pour renforcer ses compétences interpersonnelles et son estime de soi. Son objectif est d'obtenir son certificat d'études primaire du Kenya afin d'entrer à l'école secondaire du camp. Son rêve est de retourner dans son pays pour aider sa communauté. ${ }^{i}$

Nombreuses sont les filles dans le monde qui, comme Leyla, souhaitent aller à l'école et réaliser leurs rêves. Pourtant, 263 millions d'enfants, d'adolescents et de jeunes à travers le monde - soit un sur cinq - ne sont pas scolarisés (UNESCO, 2018). Bien que l'écart se réduise entre filles et garçons, en 2016, 18,5 \% des filles d'âge scolaire n'ont pas accès à une éducation, dont 35,1 \% en Afrique subsaharienne. La pauvreté, la violence, et la répartition traditionnelle des rôles au sein de la famille comptent, entre autres, au nombre des raisons pour lesquelles l'éducation des filles n'est pas priorisée partout. Pourtant, elle est essentielle à l'atteinte de l'égalité des genres et à la fondation de communautés saines et prospères (Chae et al., 2020; De Neve et al., 2015).

L'éducation des filles s'inscrit dans le cadre de la convention relative aux droits de l'enfant des Nations Unies de 1989 (AGNU, 1989). Plus spécifiquement, l'article 28 stipule que chaque enfant a droit à une éducation à tous les niveaux primaire, secondaire, supérieur. Selon l'article 29, l'éducation doit favoriser l'épanouissement de l'enfant et le développement de ses talents et de ses aptitudes. Elle doit aussi préparer l'enfant à assumer les responsabilités de la vie, dans un esprit de compréhension, de paix, de tolérance, d'égalité entre les sexes.

Plus récemment, les Nations Unies (AGNU, 2015) ont défini 17 objectifs de développement durable (ODD) 2030 qui dotent les États d'une vision commune pour mettre fin à la pauvreté, sauver la planète et bâtir un monde pacifique. En particulier, l'objectif 4 vise à assurer à tous une éducation équitable, inclusive et de qualité et des possibilités d'apprentissage tout au long de la vie. Outre l'éducation, d'autres objectifs de développement durable traitent des défis auxquels sont confrontées les filles dont 
l'autonomisation (ODD 5); la santé (ODD 3); l'accès à l'eau, l'assainissement et l'hygiène (ODD 6); le travail (ODD 8); et la protection (ODD 16).

Les études démontrent que l'éducation des filles est un investissement communautaire clé pour briser le cycle de la pauvreté. L'effet d'entraînement (« ripple effect ») est majeur comme l'illustrent les exemples suivants :

- Une année additionnelle d'éducation primaire peut accroître de $10 \%$ à $20 \%$ le salaire que les filles gagneront à l'âge adulte, et une année additionnelle au cycle secondaire peut l'augmenter de $15 \%$ à $25 \%$ (UNICEF, 2011).

- Des études sur le Kenya, le Brésil et l'Inde ont démontré que la productivité économique nationale peut augmenter respectivement de 3,4 milliards, 3,5 milliards et 7,7 milliards de dollars américains si l'on retarde la maternité chez les adolescentes (FNUAP, 2013).

- Si toutes les filles des pays à faible revenu et à revenu intermédiaire (tranche inférieure) achevaient le cycle d'éducation secondaire, la mortalité des moins de 5 ans serait réduite de moitié (UNESCO, 2014).

- Si toutes les femmes avaient accès à l'enseignement secondaire en Afrique subsaharienne ainsi qu'en Asie du Sud et de l'Ouest, le nombre des mariages d'enfants chuterait de $64 \%$ (UNESCO, 2014).

- Investir afin que les filles puissent achever le cycle d'enseignement supérieur pourrait contribuer à augmenter leurs revenus tout au long de leur vie pour représenter jusqu'à $68 \%$ du PIB annuel (UNESCO, 2014).

\section{The NOLA Project}

C'est dans ce cadre de référence international que s'inscrit The NOLA Project (http://nolaproject.org). Établi au Canada en 2014, The NOLA Project a pour but de sensibiliser les publics à l'éducation des filles avec des histoires inspirantes et des images puissantes dans un contexte d'autonomisation, d'inclusion et de réussite. Nous réalisons des reportages à forte valeur sociale pour témoigner de l'effet positif et durable qu'ont les programmes d'éducation sur la réussite des jeunes filles et sur la transformation des communautés. 
Pour réaliser nos reportages, nous collaborons avec des organismes non gouvernementaux qui sont reconnus à l'international pour leur expertise dans la mise en œuvre de programmes d'éducation destinés aux filles. Ces programmes touchent des filles marginalisées dont le parcours de vie est affecté par différents défis : des filles en situation de handicap ou de maladie, appartenant à des minorités ethniques, autochtones, migrantes, réfugiées, vivant dans des zones rurales ou urbaines.

Nos reportages documentent l'expérience positive des filles à l'école à travers des récits de vie scolaire et familiale. Inspirés par la théorie des systèmes écologiques (Bronfenbrenner, 1979, 1999), et selon une approche globale et inclusive, nous témoignons également de la contribution de toutes les parties prenantes à la réussite scolaire des filles qu'il s'agisse de la famille au sens large, des enseignants et directeurs d'école, et de la communauté dans son ensemble. Les organismes et institutions internationales à travers leurs programmes et politiques font également partie de ce cercle d'influence (Figure 1). Ces récits mettent en lumière la détermination et le courage dont font preuve les filles malgré un contexte social et économique peu favorable à la réussite scolaire. Ils racontent le rôle essentiel de toutes les personnes qui gravitent autour du cercle des filles et qui contribuent à l'accès et au maintien des filles à l'école. Ces personnes agissent comme des modèles de rôle inspirants pour permettre aux filles de développer leur plein potentiel et réaliser leurs rêves.

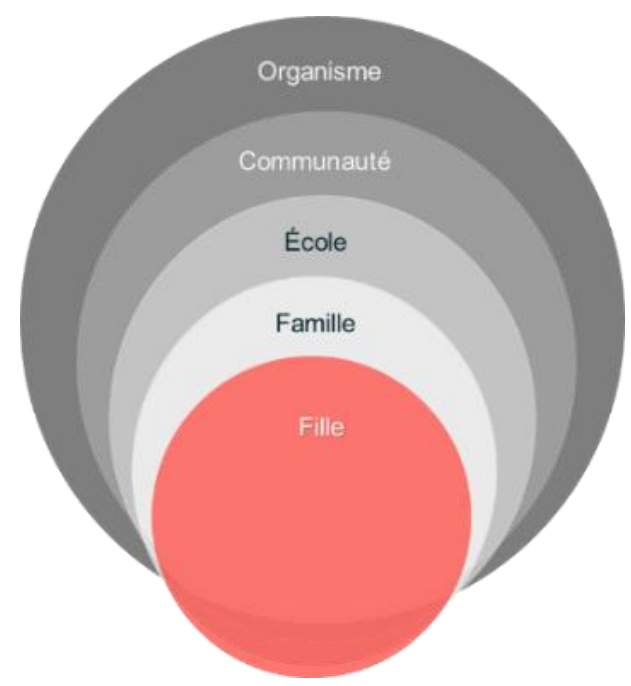

Figure 1 Approche écologique et systémique de l'éducation des filles 


\section{Angle Narratif}

À The NOLA Project, nous avons la conviction que l'éducation est le levier le plus puissant pour permettre aux filles de réaliser leur potentiel. Aussi, tous nos reportages photographiques sont construits autour du potentiel des filles. Pour documenter ces expériences à l'école, nous collectons des données qui identifient les filles, leurs familles et leurs amis. Nous voulons comprendre leur quotidien à l'école et à la maison et les interactions entre les différentes parties prenantes. Nous rencontrons également les enseignants et les directeurs d'école pour mieux identifier les facteurs qui sont mis en place pour permettre aux filles d'étudier dans un environnement apprenant, sécuritaire et qui répond à leurs besoins. Enfin, nous organisons des entretiens avec des personnes influentes de la communauté qui font la promotion du droit à l'éducation pour les filles. L'ensemble de ces données sont compilées et présentées sous forme de récits qui accompagnent les portraits. Il s'agit de récits de vie les plus exhaustifs possibles qui témoignent de l'impact de l'éducation sur la transformation de vie individuelle et collective.

En utilisant ces histoires inspirantes, nous voulons faire réfléchir les publics en leur apportant des connaissances sur l'état de l'éducation dans le monde et sur les barrières qui empêchent les filles d'aller à l'école et de s'y maintenir. Nous présentons des solutions qui sont mises en place dans les écoles, les familles, et les communautés pour assurer l'égalité des genres. Nous ne livrons pas seulement un message à un public de manière transactionnelle, mais nous cherchons plutôt à construire des ponts et à créer une communauté globale portée par un idéal commun en faveur du droit des enfants à l'éducation. 


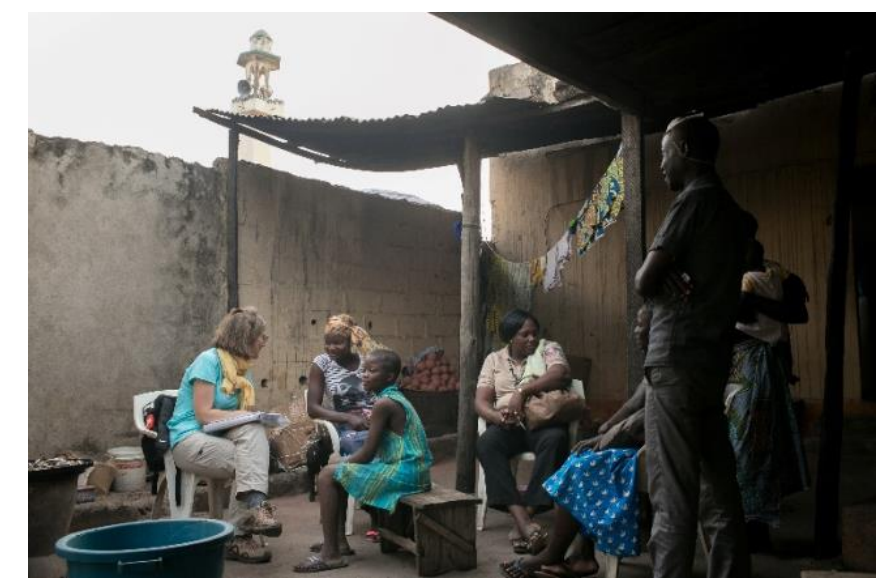

Figure 2 Entrevue avec Moussifa et les membres de sa famille dans la région de la Kara au Togo. Photographie par Dominique et Maria Cabrelli

\section{Intention Esthétique}

Nos reportages ciblent les jeunes filles et tous ceux et celles qui contribuent à a réussite scolaire des filles. Outre la visite des programmes d'éducation mis en œuvre par les organisations non gouvernementales collaboratrices, les reportages sont le reflet de rencontres enrichissantes et éblouissantes, de celles qui vous transforment et qui vous mobilisent à prendre action en faveur d'un monde plus juste et plus humain où chacun aurait sa place, où chacun pourrait réaliser son potentiel, où chacun aurait les conditions favorables pour se développer.

Nous réalisons des portraits de jeunes filles, principalement en noir et blanc en gros plan («headshot») ou en plan rapproché afin de créer une proximité avec la fille et de capter les émotions qui s'y dégagent (Figure 2). Ces portraits mettent en lumière le potentiel de chacune des jeunes filles et touchent à l'essence même de la personne, sa dignité. Au moment de l'exposition, ce type de portrait permet d'établir une connexion directe et puissante entre la fille et le spectateur. Lors de l'évaluation de la première exposition, nous avons demandé aux visiteurs leur appréciation des portraits. Certains avaient apprécié le format, les contrastes, la lumière, le reflet d'images heureuses, le sourire dans les yeux. D'autres avaient souligné que les photos donnaient l'impression que les filles étaient présentes. Outre les portraits, nous réalisons des photos de contexte qui permettent de contextualiser les filles dans leur environnement, à l'école et à la 
maison. Ces photos sont principalement en couleurs car elles permettent de mieux refléter la réalité et traduire des sensations différentes du noir et blanc. Certaines données de l'évaluation de la première exposition nous permettent de souligner que la combinaison des photos et des récits contribue à une meilleure compréhension des enjeux et des opportunités concernant l'éducation des filles. Un des répondants lors de l'évaluation nous avait dit : « Vous avez réussi à nous faire rencontrer les jeunes filles. ».

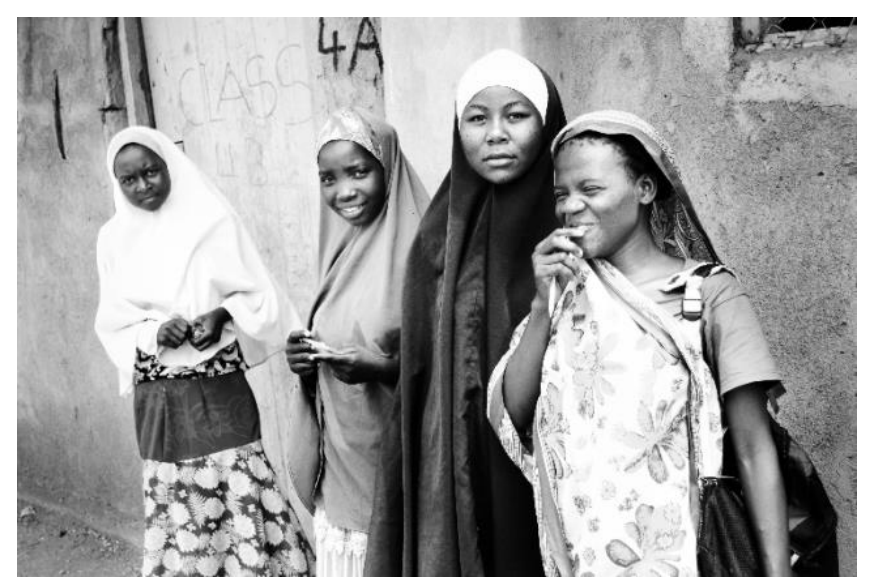

Figure 3 Filles à l'école dans le camp de réfugiés de Kakuma au Kenya. Photographie par Arvind Eyunni

\section{Considérations Éthiques}

The NOLA Project a une pratique de photographie et une technique d'entrevue respectueuses de l'enfant. Toutes les filles que nous rencontrons au cours des reportages se portent volontaires et consentent à être photographiées et interviewées. Aucun critère de sélection n'est fourni au préalable à l'organisme ou à la direction de l'école. Le directeur de l'école ou l'enseignant qui est en interaction continue avec les filles est en mesure de proposer une liste. Les filles mineures sont accompagnées d'un parent, d'un membre de leur famille ou d'un tuteur. Au début de chaque séance, nous expliquons l'objectif du reportage, ses règles, son déroulement et ses retombées, incluant les expositions. Un formulaire de consentement est utilisé pour fins d'approbation. Nous informons les filles et les accompagnants qu'une copie papier ou électronique des portraits leur seront fournis par l'intermédiaire de l'organisme, après la phase de postproduction au retour au Canada. En ce qui concerne les récits, l'organisme valide tous les 
textes et complète au besoin certaines données auprès des filles et de leurs familles. Nous rappelons aux filles qu'elles peuvent à tout moment interrompre leur participation ou ne pas répondre à certaines questions avant et pendant les séances photo et les entrevues. Cette règle s'applique également aux accompagnants. Bien que ce cas ne se soit jamais produit, il pourrait arriver qu'un participant à la séance de photo et d'entrevue ne souhaite pas que son histoire ou sa photo soit diffusée. Dans ce cas, nous respecterions son choix et nous n'utiliserions pas le matériel produit et les données collectées.

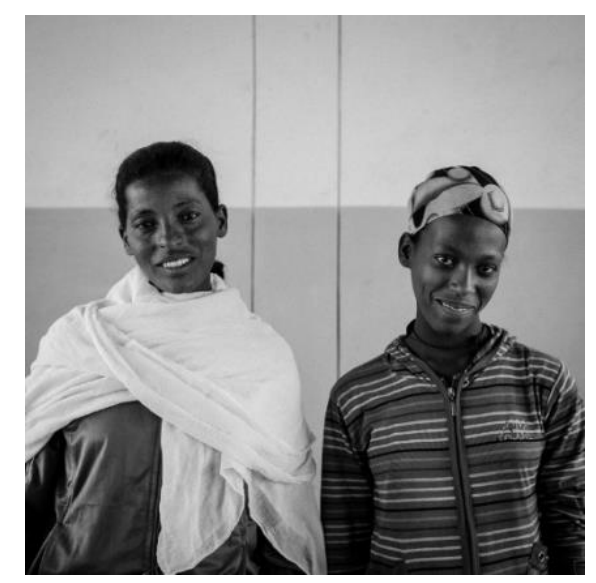

Figure 4 Fille et mère à l'école dans la communauté de Wuchale en Éthiopie. Photographie par Jean-François Lemire

\section{Sensibiliser les Publics par la Diffusion des Reportages}

Avec pour objectif de sensibiliser les publics aux droits des enfants et à l'éducation des filles, nous diffusons les reportages sur le site internet de The NOLA Project et sur les sites des collaborateurs photographes. Les organismes peuvent également utiliser le matériel pour fins de communication auprès de leur communauté de donateurs et partenaires. Enfin, pour permettre à un plus large auditoire de vivre une expérience unique et immersive dans un contexte multiculturel et de développement international, nous présentons les reportages sous forme d'expositions dans des institutions internationales qui ont une mission éducative auprès des publics. C'est le cas notamment du Musée des beaux-arts de Montréal qui possède le plus grand complexe éducatif dans un musée d'art en Amérique. 


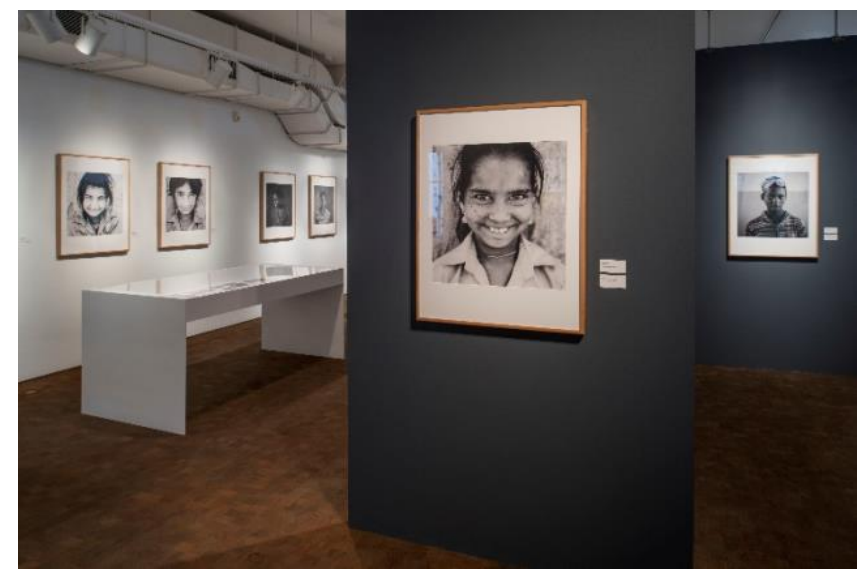

Figure 5 Exposition de portraits de filles au Musée des beaux-arts de Montréal. Photographie par le Musée des beaux-arts de Montréal

C'est ainsi qu'est née l'exposition «Quand je serai grande, je serai... Pour une éducation inclusive des filles d'ici et d'ailleurs », en collaboration avec Humanité \& Inclusion, Help Lesotho et The Woman Power. Cette exposition a été présentée du 9 octobre 2018 au 20 janvier 2019 à l'espace d'exposition J.A. DeSève de l'Atelier international d'éducation et d'art-thérapie Michel de la Chenelière au Musée des beauxarts de Montréal. ${ }^{\text {ii }}$

Accompagnés par l'équipe du Musée, nous avons choisi de construire l'exposition sous l'angle du potentiel des jeunes filles et de leurs rêves, rendus possibles grâce à une éducation de qualité et à des parties prenantes qui agissent comme des modèles de rôle (familles, corps enseignants, chefs de file communautaires, etc.). L'ancrage dans la réalité montréalaise nous est apparu essentiel pour créer une plus grande proximité avec les visiteurs et illustrer l'universalité du propos. Enfin, nous avons sélectionné une œuvre de la collection du Musée qui servirait de point de départ à cette présentation. Ce face-à-face entre Togolaises, Lesothanes et Montréalaises a permis de jeter un pont entre les filles du monde et de lancer une conversation universelle sur l'éducation comme levier de développement individuel et collectif. Ces parcours aussi diversifiés qu'uniques mettent 
en lumière le rôle fondamental des modèles féminins comme sources d'inspiration et de dépassement de soi.

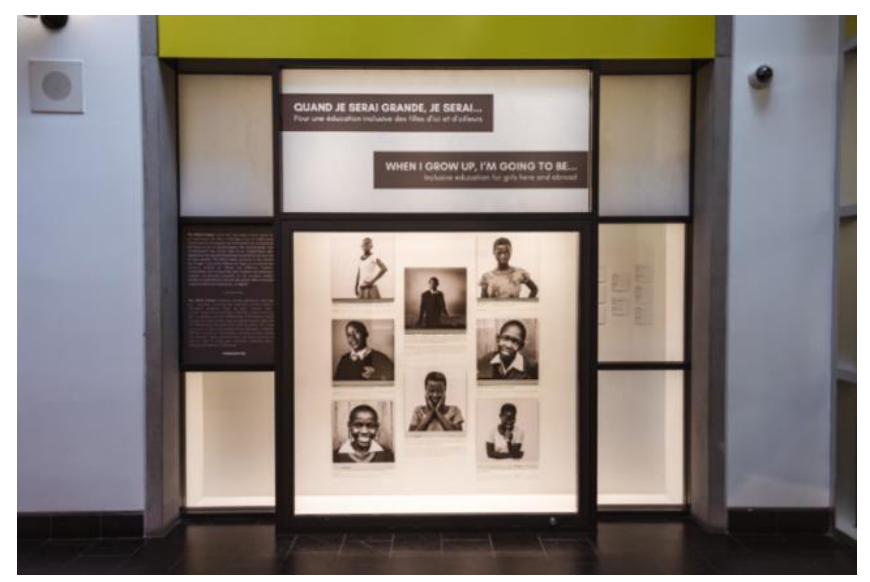

Figure 6 Exposition de portraits de filles au Musée des beaux-arts de Montréal. Photographie par le Musée des beaux-arts de Montréal

Afin de rejoindre un public global engagé dans la promotion des droits des femmes, The NOLA Project a présenté l'exposition à la conférence Women Deliver 2019, qui s'est tenue en juin 2019 à Vancouver. Il s'agissait de la plus importante conférence internationale du vingt et unième siècle consacrée aux droits des femmes et à l'égalité des genres. Caisse de résonnance amplifiant la voix des jeunes filles, l'exposition a naturellement trouvé sa place sur cette tribune internationale.

Une autre étape majeure pour The NOLA Project sera la présentation d'une version alternative de l'exposition au tout nouveau Lesotho National Museum and Art Gallery, à Maseru, en 2021, et ce à la demande de l'équipe de développement du futur musée. Il s'agit d'une occasion exceptionnelle de célébrer la réussite des jeunes filles de la communauté de Leribe (Lesotho) dans leur propre pays. 


\section{Conclusion}

À travers des images puissantes et des récits inspirants sur l'éducation des filles vulnérables dans le monde, The NOLA Project se positionne en défenseur des droits des enfants et du droit à l'éducation, un droit fondamental inter-relié aux autres droits humains.

Finalement Leyla a réussi son certificat et obtenu une bourse pour étudier au secondaire dans une école nationale au Kenya en dehors du camp de réfugiés.

Leyla est cette voix inaudible qui résonne dans le vibrant plaidoyer en faveur de l'éducation pour tous prononcé par Malala Yousafzai à l'ONU le 12 juillet 2013 : « Je ne parle pas en mon nom mais au nom de tous ceux dont la voix ne peut être entendue. Au nom de tous ceux qui luttent pour leur droit de vivre dans la paix, leur droit d'être traité avec dignité, leur droit à l'égalité des chances et leur droit à l'éducation. » (PNUD, 2013).

Remerciements : Nous tenons à remercier tous ceux et celles qui nous ont aidés à la réalisation de ce projet, notamment les filles et leurs familles qui ont participé aux reportages photographiques, les corps enseignants et les directions d'écoles, ainsi que les organismes non gouvernementaux partenaires. Nous exprimons toute notre gratitude à Dr. Mónica Ruiz-Casares pour son conseil, particulièrement dans la conduite éthique de la recherche auprès des enfants. Enfin, nous remercions la direction du Musée des beaux-arts de Montréal qui a favorisé le rayonnement du projet auprès du public. 


\section{Références}

AGNU. (1989). Convention relative aux droits de l'enfant des Nations Unies. Rés A.G. 44/25, Doc. N.U. A/44/49. New York: Assemblée Générale des Nations Unies. Disponible 28 février 2020 : https://www.ohchr.org/fr/professionalinterest/pages/crc.aspx

AGNU. (2015). Transformer notre monde : le Programme de développement durable à 1'horizon 2030. Rés A.G. du 25 septembre 2015, Doc. N.U. A/RES/70/1. New York: Assemblée Générale des Nations Unies. Disponible 28 février 2020 : https://undocs.org/fr/A/RES/70/1

Bronfenbrenner, U. (1979). The ecology of human development. Cambridge, MA: Harvard University Press.

Bronfenbrenner, U. (1999). Environments in Developmental Perspective: Theoretical and Operational Models. In S. L. Friedman \& T. D. Wachs (Eds.), Measuring Environment across the Life Span: Emerging Methods and Concepts (pp. 3-28). Washington, DC: American Psychological Association.

Chae, S., Haberland, N., McCarthy, K. J., Weber, A. M., Darmstadt, G. L., \& Ngo, T. D. (2020). The Influence of Schooling on the Stability and Mutability of Gender Attitudes: Findings From a Longitudinal Study of Adolescent Girls in Zambia. Journal of Adolescent Health, 66(1), S25-S33. doi:10.1016/j.jadohealth.2019.08.031

De Neve, J.-W., Fink, G., Subramanian, S. V., Moyo, S., \& Bor, J. (2015). Length of secondary schooling and risk of HIV infection in Botswana: evidence from a natural experiment. The Lancet Global Health, 3(8), e470-e477. doi:10.1016/S2214-109X(15)00087-X

FNUAP. (2013). État de la population mondiale : La mère-enfant : Face aux défis de la grossesse chez l'adolescente. New York: Fonds des Nations Unies pour la population Disponible 28 février 2020 : http://www.unfpa.org/sites/default/files/pub-pdf/FR-SWOP2013.pdf

PNUD. (2013). ONU : vibrant plaidoyer de Malala Yousafzai en faveur de l'éducation pour tous. 
https://www.undp.org/content/undp/fr/home/presscenter/articles/2013/07/12/atun-malala-yousafzai-rallies-youth-to-stand-up-for-universal-education.html

UNESCO. (2014). Rapport mondial de suivi sur l'EPT : Enseigner et apprendre :

Atteindre la qualité pour tous. Paris. Disponible 28 février 2020 :

https://unesdoc.unesco.org/ark:/48223/pf0000226157

UNESCO. (2018). «One in Five Children, Adolescents and Youth is Out of School», Fact

Sheet no 48, février 2018. Disponible 28 février 2020 :

http://uis.unesco.org/sites/default/files/documents/fs48-one-five-childrenadolescents-youth-out-school-2018-en.pdf

UNICEF. (2011). Nations Unies, Groupe de travail interinstitutionnel sur les femmes rurales, Fact Sheet: Rural Women and the Millennium Development Goals et UNICEF (2011). Disponible 28 février 2020 :

http://www.unicef.org/media/media_58417.html

Notes

i Extrait d'une entrevue réalisée par The NOLA Project en mai 2012 dans le camp de réfugiés de Kakuma avec la collaboration des organismes Entraide universitaire mondiale du Canada et Windle International Kenya. Suivi du programme réalisé en 2014.

ii Article « Pour une éducation inclusive des filles d'ici et d'ailleurs - Un projet qui rayonne à l'international » paru dans le magazine $\mathrm{M}$ du Musée des beaux-arts de Montréal septembre-décembre 2019. 